\title{
Some Fixed Point Results of Ciric-Type Contraction Mappings on Ordered $G$-Partial Metric Spaces
}

\author{
Johnson O. Olaleru' ${ }^{1}$, Kanayo Stella Eke ${ }^{2}$, Hallowed O. Olaoluwa1 \\ ${ }^{1}$ Department of Mathematics, University of Lagos, Akoka, Lagos, Nigeria \\ ${ }^{2}$ Department of Mathematics, Covenant University, Ota, Nigeria \\ Email: olaleru1@yahoo.co.uk, ugbohstella@yahoo.com, olu20_05@hotmail.com
}

Received 2 November 2013; revised 7 December 2013; accepted 25 December 2013

Copyright (C) 2014 by authors and Scientific Research Publishing Inc.

This work is licensed under the Creative Commons Attribution International License (CC BY). http://creativecommons.org/licenses/by/4.0/

c) (i) Open Access

\begin{abstract}
We introduce the concept of generalized quasi-contraction mappings in $G$-partial metric spaces and prove some fixed point results in ordered $G$-partial metric spaces. The results generalize and extend some recent results in literature.
\end{abstract}

\section{Keywords}

Fixed Points, Generalized Quasi-Contraction Maps, Bounded Orbit, Partially Ordered Set, $G$-Partial Metric Spaces

\section{Introduction and Preliminary Definitions}

The Banach contraction principle has been generalized and extended in many directions for some decades. Of all the generalizations, Ciric [1] [2] generalizations seem outstanding. Cho Song Wong [3] dealt with a pair of operators in which the control functions in the generalized contraction maps are upper semi-continuous, while Ciric considered a single operator and took the control function to be a constant. If the control function is an upper semi-continuous, then the result of Ciric [1] is invalid. In Kiany and Amini-Harandi [4], a condition is imposed on the control function and the mapping is termed a Ciric generalized quasi-contraction mapping. In this work, we introduce the concept of generalized quasi-contraction mappings in the new framework of $G$-partial metric spaces.

Rodriguez-Lopez and Nieto [5], Ran and Reuring [6] presented some new results for the existence of the fixed point for some mappings in partially ordered metric spaces. The main idea in [5] [6] involves combing the ideas of an iterative technique in the contraction mapping principle with those in the monotone technique. In this 
work, the existence of a unique fixed point for generalized contraction mappings in ordered $G$-partial metric spaces is proved.

Matthew [7] generalized the notion of metric spaces by introducing the concept of nonzero self-distance and thus, defined a generalized metric space known as partial metric space, as follows:

Definition 1.1. [7]. A partial metric space is a pair $(X, p)$, where $X$ is a nonempty set and $p: X \times X \rightarrow \mathbb{R}$ such that:

(p1) $0 \leq p(x, x) \leq p(x, y)$

(p2) if $p(x, x)=p(x, y)=p(y, y)$, then $x=y$

(p3) $p(x, y)=p(y, x)$

(p4) $p(x, z) \leq p(x, y)+p(y, z)-p(y, y)$.

He was able to establish a relationship between partial metric spaces and the usual metric spaces when

$$
d p(x, y)=2 p(x, y)-p(x, x)-p(y, y) .
$$

Mustafa and Sims [8] also extended the concepts of metric to $G$-metric by assigning a positive real number to every triplet of an arbitrary set as follows:

Definition 1.2. [8]. Let $X$ be a nonempty set, and let

$G: X \times X \times X \rightarrow \mathbb{R}_{+}$be a function satisfying:

(G1) $G(x, y, z)=0$ if $x=y=z$,

(G2) $0<G(x, x, y)$ for all $x, y \in X$ with $x \neq y$,

(G3) $G(x, x, y) \leq G(x, y, z)$ for all $x, y, z \in X$ with $y \neq z$,

(G4) $G(x, y, z)=G(x, z, y)=G(y, z, x)$ (symmetry in all three variables),

(G5) $G(x, y, z) \leq G(x, a, a)+G(a, y, z)$ for all $x, y, z, a \in X$ (rectangle inequality).

Then, the function $G$ is called a generalized metric, or more specifically, a $G$-metric on $X$, and the pair $(X, G)$ is a $G$-metric space.

Mustafa [8] gave an example to show the relationship between $G$-metric spaces and ordinary metric spaces as: For any $G$-metric $G$ on $X$, if $d_{G}(x, y)=G(x, y, y)+G(x, x, y)$, then $\left(X, d_{G}\right)$ is a metric space.

In this work, the idea of the nonzero self-distance of partial metric spaces and the rectangle inequality of $G$-metric spaces are combined to develop a new generalized metric space which is defined as the following:

Definition 1.3. Let $X$ be a nonempty set, and let $G_{p}: X \times X \times X \rightarrow \mathbb{R}_{+}$be a function satisfying the following:

(Gp1) $G_{p}(x, y, z) \geq G_{p}(x, x, x) \geq 0, \forall x, y, z \in X \quad$ (small self-distance),

(Gp2) $G_{p}(x, y, z)=G_{p}(x, x, y)=G_{p}(y, y, z)=G_{p}(z, z, x)$ iff $x=y=z$, (equality),

(Gp3) $G_{p}(x, y, z)=G_{p}(z, x, y)=G_{p}(y, z, x)$ (symmetry in all three variables),

(Gp4) $G_{p}(x, y, z) \leq G_{p}(x, a, a)+G_{p}(a, y, z)-G_{p}(a, a, a)$ (Rectangle inequality).

The function $G_{p}$ is called a $G$-partial metric and the pair $\left(X, G_{p}\right)$ is called a $G$-partial metric space.

Definition 1.4. A $G$-partial metric space is said to be symmetric if $G_{p}(x, y, y)=G_{p}(y, x, x)$ for all $x, y \in X$.

In this work, we will assume that $\left(X, G_{p}\right)$ is symmetric. The following proposition establishes the relation between $G$-partial metric spaces and (partial) metric spaces.

Definition 1.5. Let $\left(X, G_{p}\right)$ be a $G$-partial metric space. Define the functions $p: X \times X \rightarrow \mathbb{R}_{+}$and $d: X \times X \rightarrow \mathbb{R}_{+}$by $p(x, y)=G_{p}(x, y, y)+G_{p}(y, x, x)$ and $d(x, y)=G_{p}(x, y, y)+G_{p}(y, x, x)-G_{p}(y, y, y)-G_{p}(x, x, x)$. Then

1) $(X, p)$ is a partial metric space.

2) $(X, d)$ is a metric space.

Proof

1) From (Gp1), we have that for all $x, y \in X$,

$$
p(x, y)=G p(x, y, y)+G p(y, x, x) \geq G p(x, x, x)+G p(x, x, x)=p(x, x) \geq 0,
$$

hence $(p 1)$ is satisfied.

If $p(x, x)=p(y, y)=p(y, y)$, then

$$
G p(x, x, x)+G p(x, x, x)=G p(x, y, y)+G p(y, x, x)=G p(y, y, y)+G p(y, y, y) \text {. }
$$

By (Gp1), it must follow that $G p(x, y, y)=G p(y, x, x)=G p(y, y, y)=G p(x, x, x)$. 
From the symmetry of $G_{p}$ and by $(G p 2), x=y$, hence $(p 2)$ is satisfied.

(p3) follows from (Gp3) and the triangle inequality ( $p 4)$ is easily verifiable using $(G p 4)$.

2) Since $(X, p)$ is a partial metric space, then

$$
p^{s}=2 p(x, y)-p(x, x)-p(y, y)=2[G p(x, y, y)+G p(y, x, x)-G p(y, y, y)-G p(x, x, x)]
$$

defines a metric on $X$ and so $d(x, y)=\frac{1}{2} p^{s}(x, y)$ also defines a metric on $X$.

Example 1.6. Let $X=\mathbb{R}_{+}$and define the function $G p: X \times X \times X \rightarrow R_{+}$as $G p(x, y, z)=\max \{x, y, z\}$. Then $\left(X, G_{p}\right)$ is a $G$-partial metric space.

We state the following definitions and motivations.

Definition 1.7. A sequence $\left\{x_{n}\right\}$ of points in a $G$-partial metric space $\left(X, G_{p}\right)$ converges to some $a \in X$ if $\lim _{n \rightarrow \infty} G p\left(x_{n}, x_{n}, a\right)=\lim _{n \rightarrow \infty} G p\left(x_{n}, x_{n}, x_{n}\right)=G p(a, a, a)$.

Definition 1.8. A sequence $\left\{x_{n}\right\}$ of points in a $G$-partial metric spaces $\left(X, G_{p}\right)$ is Cauchy if the numbers $G_{p}\left(x_{n}, x_{m}, x_{l}\right)$ converges to some $a \in X$ as $n, m, l$ approach infinity.

The proof of the following result follows from the above definitions:

Proposition 1.9. Let $\left\{x_{n}\right\}$ be a sequence in $G$-partial metric space $X$ and $a \in X$. If $\left\{x_{n}\right\}$ converges to $a \in X$ then $\left\{x_{n}\right\}$ is a Cauchy sequence.

Definition 1.10. A $G$-partial metric space $\left(X, G_{p}\right)$ is said to be complete if every Cauchy sequence in $\left(X, G_{p}\right)$ converges to an element in $\left(X, G_{p}\right)$.

Definition 1.11. [6]. If $(X, \prec)$ is a partially ordered set and $T: X \rightarrow X$, then $T$ is monotone non-decreasing if for every $x, y \in X, \quad x \prec y$ implies $T x \prec T y$.

Definition 1.12. Let $(X, \prec)$ be a partially ordered set. Then two elements $x, y \in X$ are said to be totally ordered or ordered if they are comparable, i.e. $x \prec y$ or $y \prec x$.

Gordji et al. [9] proved the existence of a unique fixed point for contraction type maps in partially ordered metric spaces using a control function. Kiany and Amini-Harandi [4] proved the existence of a unique fixed point for a generalized Ciric quasi-contraction mapping in what they tagged a generalized metric space. The map they considered extend that of Gordji et al., albeit the space they considered was not endowed with an order. Saadati et al. [10] considered the concept of Omega-distances on a complete partially ordered G-metric space and proved some fixed point theorems. Turkoglu et al. [11] and Sastry et al. [12] proved some fixed point theorems for generalized contraction mappings in cone metric spaces and metric spaces respectively.

In this work, the existence of unique fixed points of the two generalized contraction mappings below is proved in ordered $G$-partial metric spaces, extending thus the results in [2] [4] [9] [11].

Definition 1.13. Let $\left(X, G_{p}\right)$ be a $G$-partial metric space. The self-map $T: X \rightarrow X$ is said to be a generalized Ciric quasi-contraction if

$$
G_{p}(T x, T y, T y) \leq \alpha(x, y, y) \max \left\{G_{p}(x, y, y), G_{p}(x, T x, T x), G_{p}(y, T y, T y), G_{p}(x, T y, T y), G_{p}(y, T x, T x)\right\}
$$

for any $x, y \in X$, where $\alpha:[0, \infty) \rightarrow[0,1)$ is a mapping.

Definition 1.14. Let $\left(X, G_{p}\right)$ be a $G$-partial metric space. The self-map $T: X \rightarrow X$ is said to be a generalized $G$-contraction if for all $x, y \in X$,

$$
\begin{aligned}
& G_{p}(T x, T y, T y) \leq \alpha(x, y, y) G_{p}(x, y, y)+\beta(x, y, y) G_{p}(x, T x, T x) \\
& +\gamma(x, y, y) G_{p}(y, T y, T y)+\delta(x, y, y)\left[G_{p}(x, T y, T y)+G_{p}(y, T x, T x)\right],
\end{aligned}
$$

where $\alpha, \beta, \gamma, \delta: X \times X \rightarrow[0,1)$ are functions such that

$$
\lambda=\sup \{\alpha(x, y, y)+\beta(x, y, y)+\gamma(x, y, y)+2 \delta(x, y, y): x, y \in X\}<1 \text {. }
$$

\section{Main Results}

Theorem 2.1. Let $(X, \prec)$ be a partially ordered set and suppose there exists a $G$-partial metric $G_{p}$ in $X$ such that $\left(X, G_{p}\right)$ is a complete $G$-partial metric space. Let $T: X \rightarrow X$ be a self-mapping in $X$ such that for each 
$x, y \in X$ satisfying $x \prec y$,

$$
\begin{aligned}
& G_{p}(T x, T y, T y) \leq \alpha(x, y, y) G_{p}(x, y, y)+\beta(x, y, y) G_{p}(x, T x, T x) \\
& +\gamma(x, y, y) G_{p}(y, T y, T y)+\delta(x, y, y)\left[G_{p}(x, T y, T y)+G_{p}(y, T x, T x)\right],
\end{aligned}
$$

where $\alpha, \beta, \gamma, \delta: X \times X \rightarrow[0,1)$ are functions such that

$$
\lambda=\sup \{\alpha(x, y, y)+\beta(x, y, y)+\gamma(x, y, y)+2 \delta(x, y, y): x, y \in X\}<1 .
$$

Suppose $T$ is a non-decreasing map such that there exists an $x_{0} \in X$ with $x_{0} \prec T x_{0}$. Also suppose that $X$ is such that for any non-decreasing sequence $\left\{x_{n}\right\}$ converging to $x, \quad x_{n} \prec x, \forall n \in \mathbb{N}$. $\quad x_{n} \prec x$ for all $n \in \mathbb{N}$.

Then $T$ has a fixed point. Moreover, if for each $u, v \in X$, there exists $z \in X$ which is comparable to $u$ and $v$, then $T$ has a unique fixed point.

Proof. Fix $x_{0} \in X$ Let $\left\{x_{n}\right\}$ be defined by $x_{1}=T x_{0}, x_{2}=T x_{1}, \cdots, x_{n+1}=T x_{n}$. Since $x_{0} \prec T x_{0}$ and $T$ is non-decreasing, then $x_{0} \prec T x_{0} \prec T x_{1} \prec T x_{2} \prec \cdots \prec T x_{n} \prec \cdots$

This implies that $x_{n} \prec T x_{n}$ for each $n \geq 1$.

Since $x_{n} \prec x_{n+1}$ for each $n \in \mathbb{N}$ then by (3) we have

$$
\begin{aligned}
& G_{p}\left(x_{n}, x_{n+1}, x_{n+1}\right)=G_{p}\left(T x_{n-1}, T x_{n}, T x_{n}\right) \\
& \leq \\
& \quad \alpha\left(x_{n-1}, x_{n}, x_{n}\right) G_{p}\left(x_{n-1}, x_{n}, x_{n}\right)+\beta\left(x_{n-1}, x_{n}, x_{n}\right) G_{p}\left(x_{n-1}, T x_{n-1}, T x_{n-1}\right)+\gamma\left(x_{n-1}, x_{n}, x_{n}\right) G_{p}\left(x_{n}, T x_{n}, T x_{n}\right) \\
& \quad+\delta\left(x_{n-1}, x_{n}, x_{n}\right)\left[G_{p}\left(x_{n-1}, T x_{n}, T x_{n}\right)+G_{p}\left(x_{n}, T x_{n-1}, T x_{n-1}\right)\right] \\
& \leq \alpha\left(x_{n-1}, x_{n}, x_{n}\right) G_{p}\left(x_{n-1}, x_{n}, x_{n}\right)+\beta\left(x_{n-1}, x_{n}, x_{n}\right) G_{p}\left(x_{n-1}, x_{n}, x_{n}\right)+\gamma\left(x_{n-1}, x_{n}, x_{n}\right) G_{p}\left(x_{n}, x_{n+1}, x_{n+1}\right) \\
& \quad+\delta\left(x_{n-1}, x_{n}, x_{n}\right)\left[G_{p}\left(x_{n-1}, x_{n+1}, x_{n+1}\right)+G_{p}\left(x_{n}, x_{n}, x_{n}\right)\right] \\
& \leq \alpha\left(x_{n-1}, x_{n}, x_{n}\right) G_{p}\left(x_{n-1}, x_{n}, x_{n}\right)+\beta\left(x_{n-1}, x_{n}, x_{n}\right) G_{p}\left(x_{n-1}, x_{n}, x_{n}\right)+\gamma\left(x_{n-1}, x_{n}, x_{n}\right) G_{p}\left(x_{n}, x_{n+1}, x_{n+1}\right) \\
& \quad+\delta\left(x_{n-1}, x_{n}, x_{n}\right)\left[G_{p}\left(x_{n-1}, x_{n}, x_{n}\right)+G_{p}\left(x_{n}, x_{n+1}, x_{n+1}\right)-G_{p}\left(x_{n}, x_{n}, x_{n}\right)+G_{p}\left(x_{n}, x_{n}, x_{n}\right)\right] \\
& \leq \alpha\left(x_{n-1}, x_{n}, x_{n}\right) G_{p}\left(x_{n-1}, x_{n}, x_{n}\right)+\beta\left(x_{n-1}, x_{n}, x_{n}\right) G_{p}\left(x_{n-1}, x_{n}, x_{n}\right)+\gamma\left(x_{n-1}, x_{n}, x_{n}\right) G_{p}\left(x_{n}, x_{n+1}, x_{n+1}\right) \\
&+\delta\left(x_{n-1}, x_{n}, x_{n}\right)\left[G_{p}\left(x_{n-1}, x_{n}, x_{n}\right)+G_{p}\left(x_{n}, x_{n+1}, x_{n+1}\right)\right] .
\end{aligned}
$$

Thus, with $\alpha, \beta, \gamma, \delta$ evaluated at $\left(x_{n-1}, x_{n}, x_{n}\right)$, we have

$$
\begin{aligned}
G_{p}\left(x_{n}, x_{n+1}, x_{n+1}\right) \leq & \alpha G_{p}\left(x_{n-1}, x_{n}, x_{n}\right)+\beta G_{p}\left(x_{n-1}, x_{n}, x_{n}\right)+\gamma G_{p}\left(x_{n}, x_{n+1}, x_{n+1}\right) \\
& +\delta\left[G_{p}\left(x_{n-1}, x_{n}, x_{n}\right)+G_{p}\left(x_{n}, x_{n+1}, x_{n+1}\right)\right] \\
\leq & (\alpha+\beta+\gamma) \max \left\{G_{p}\left(x_{n-1}, x_{n}, x_{n}\right), G_{p}\left(x_{n}, x_{n+1}, x_{n+1}\right)\right\} \\
& +2 \delta \max \left\{G_{p}\left(x_{n-1}, x_{n}, x_{n}\right), G_{p}\left(x_{n}, x_{n+1}, x_{n+1}\right)\right\} \\
\leq & (\alpha+\beta+\gamma+2 \delta) \max \left\{G_{p}\left(x_{n-1}, x_{n}, x_{n}\right), G_{p}\left(x_{n}, x_{n+1}, x_{n+1}\right)\right\} \\
\leq & \lambda \max \left\{G_{p}\left(x_{n-1}, x_{n}, x_{n}\right), G_{p}\left(x_{n}, x_{n+1}, x_{n+1}\right)\right\} .
\end{aligned}
$$

Since $\lambda<1$, then (5) becomes $G_{p}\left(x_{n}, x_{n+1}, x_{n+1}\right) \leq \lambda G_{p}\left(x_{n-1}, x_{n}, x_{n}\right)$.

Consequently, $G_{p}\left(x_{n}, x_{n+1}, x_{n+1}\right) \leq \lambda^{n} G_{p}\left(x_{0}, x_{1}, x_{1}\right)$.

For $m>n$ we get,

$$
\begin{aligned}
& G_{p}\left(x_{n}, x_{m}, x_{m}\right) \leq G_{p}\left(x_{n}, x_{m-1}, x_{m-1}\right)+G_{p}\left(x_{m-1}, x_{m}, x_{m}\right)-G_{p}\left(x_{m-1}, x_{m-1}, x_{m-1}\right) \\
& \leq G_{p}\left(x_{n}, x_{m-2}, x_{m-2}\right)+G_{p}\left(x_{m-2}, x_{m-1}, x_{m-1}\right)-G_{p}\left(x_{m-2}, x_{m-2}, x_{m-2}\right)+G_{p}\left(x_{m-1}, x_{m}, x_{m}\right)-G_{p}\left(x_{m-1}, x_{m-1}, x_{m-1}\right) \\
& \leq \cdots \leq G_{p}\left(x_{n}, x_{n+1}, x_{n+1}\right)+G_{p}\left(x_{n+1}, x_{n+2}, x_{n+2}\right)-G_{p}\left(x_{n+1}, x_{n+1}, x_{n+1}\right) \\
& \quad+G_{p}\left(x_{n+2}, x_{n+3}, x_{n+3}\right)-G_{p}\left(x_{n+2}, x_{n+2}, x_{n+2}\right)+\cdots+G_{p}\left(x_{m-1}, x_{m}, x_{m}\right)-G_{p}\left(x_{m-1}, x_{m-1}, x_{m-1}\right) \\
& \leq \lambda^{n}\left(1+\lambda+\cdots+\lambda^{m-n}\right) G_{p}\left(x_{0}, x_{1}, x_{1}\right) \leq \lambda^{n}(1+\lambda+\cdots) G_{p}\left(x_{0}, x_{1}, x_{1}\right) \leq \frac{\lambda^{n}}{1-\lambda} G_{p}\left(x_{0}, x_{1}, x_{1}\right) .
\end{aligned}
$$


Take the limit as $n \rightarrow \infty$ in (6) yields $\lim G_{p}\left(x_{n}, x_{m}, x_{m}\right)=0$ which implies that $\left\{x_{n}\right\}$ is a Cauchy sequence. Since $X$ is a complete space then theré exists $x \in X$ such that $\left\{x_{n}\right\}$ converges to $x$ and

$$
\lim _{n \rightarrow \infty} G_{p}\left(x_{n}, x, x\right)=\lim _{n, m \rightarrow \infty} G_{p}\left(x_{n}, x_{n}, x_{n}\right)=G_{p}(x, x, x)=\lim _{n, m \rightarrow \infty} G_{p}\left(x_{n}, x_{m}, x_{m}\right)=0 .
$$

Next we prove that $x$ is the fixed point of $T$. From (3) and (4), since $x_{n} \prec x$, for all $n \in \mathbb{N}$,

$$
\begin{aligned}
& G_{p}(x, T x, T x) \leq G_{p}\left(x, x_{n}, x_{n}\right)+G_{p}\left(x_{n}, T x, T x\right)-G_{p}\left(x_{n}, x_{n}, x_{n}\right) \leq G_{p}\left(x, x_{n}, x_{n}\right)+G_{p}\left(T x_{n-1}, T x, T x\right) \\
& \leq G_{p}\left(x, x_{n}, x_{n}\right)+\alpha G_{p}\left(x_{n-1}, x, x\right)+\beta G_{p}\left(x_{n-1}, T x_{n-1}, T x_{n-1}\right)+\gamma G_{p}(x, T x, T x) \\
& \quad+\delta\left[G_{p}\left(x_{n-1}, T x, T x\right)+G_{p}\left(x, T x_{n-1}, T x_{n-1}\right)\right],
\end{aligned}
$$

where $\alpha, \beta, \gamma, \delta$ are evaluated at $\left(x, x_{n-1}, x_{n-1}\right)$.

Take limit as $n \rightarrow \infty$ yields

$$
G_{p}(x, T x, T x) \leq \gamma G_{p}(x, T x, T x)+\delta G_{p}(x, T x, T x) \leq(\gamma+\delta) G_{p}(x, T x, T x) \leq \lambda G_{p}(x, T x, T x) .
$$

Since $\lambda<1$, then $G_{p}(x, T x, T x)=0$. Hence $x=T x$.

For uniqueness, suppose $u, v \in X$ and $u \neq v$ are two fixed points of $T$, and there exists $z \in X$ which is comparable to $u$ and $v$. Monotonicity of $T$ implies that $T z_{n}$ is comparable to $T^{n} u=u$ and $T^{n} v=v$ for $n=0,1,2, \cdots$.

Moreover

$$
\begin{aligned}
& G_{p}\left(T^{n} z, T^{n} u, T^{n} u\right) \leq \alpha G_{p}\left(T^{n-1} z, u, u\right)+\beta G_{p}\left(T^{n-1} z, T^{n} z, T^{n} z\right)+\gamma G_{p}\left(u, T^{n} u, T^{n} u\right) \\
& \quad+\delta\left[G_{p}\left(T^{n-1} z, T^{n} u, T^{n} u\right)+G_{p}\left(u, T^{n} z, T^{n} z\right)\right] \\
& \leq \alpha G_{p}\left(T^{n-1} z, u, u\right)+\beta G_{p}\left(T^{n-1} z, T^{n} z, T^{n} z\right)+\gamma G_{p}\left(u, T^{n} u, T^{n} u\right) \\
& \quad+\delta\left[G_{p}\left(T^{n-1} z, T^{n} u, T^{n} u\right)+G_{p}\left(u, T^{n-1} z, T^{n-1} z\right)+G_{p}\left(T^{n-1} z, T^{n} z, T^{n} z\right)-G_{p}\left(\left(T^{n-1} z, T^{n-1} z, T^{n-1} z\right)\right]\right.
\end{aligned}
$$

where $\alpha, \beta, \gamma, \delta$ are evaluated at $\left(T^{n-1} z, T^{n-1} u, T^{n-1} u\right)$.

Taking the limit as $n \rightarrow \infty$ and by symmetry we get,

$$
G_{p}\left(T^{n} z, u, u\right) \leq \alpha G_{p}\left(T^{n-1} z, u, u\right)+2 \delta G_{p}\left(T^{n-1} z, u, u\right) \leq(\alpha+2 \delta) G_{p}\left(T^{n-1} z, u, u\right) \leq \lambda G_{p}\left(T^{n-1} z, u, u\right)
$$

Consequently, $G_{p}\left(T^{n} z, u, u\right) \leq \lambda^{n} G_{p}\left(T z_{0}, u, u\right)$.

Similarly, $G_{p}\left(T^{n} z, v, v\right) \leq \lambda^{n} G_{p}\left(T z_{0}, v, v\right)$.

Finally for all $n \in \mathbb{N}$ with $n \geq \tau$ where $\tau \in \mathbb{N}$ we have,

$$
\begin{aligned}
G_{p}(u, v, v) & \leq G_{p}\left(u, T^{n-1} z, T^{n-1} z\right)+G_{p}\left(T^{n-1} z, v, v\right)-G_{p}\left(T^{n-1} z, T^{n-1} z, T^{n-1} z\right) \\
& \leq \lambda^{n-\tau} G_{p}\left(u, T^{\tau} z_{0}, T^{\tau} z_{0}\right)+\lambda^{n-\tau} G_{p}\left(T^{\tau} z_{0}, v, v\right) .
\end{aligned}
$$

Letting $n \rightarrow \infty$ yields $G_{p}(u, v, v)=0$. Hence $u=v$.

Theorem 2.1 can be viewed as an extension of results of Turkoglu et al. ([11], Theorem 2.1) to the setting of $G$-partial metric spaces endowed with an order. The following corollary can be obtained:

Corollary 2.2. Let $(X, \prec)$ be a partially ordered set and let there exist a $G$-partial metric $G_{p}$ in $X$ such that $\left(X, G_{p}\right)$ is a complete $G$-partial metric space. Let $T: X \rightarrow X$ be a self-mapping in $X$ such that for each $x, y \in X$ satisfying $x \prec y$,

$$
G_{p}(T x, T y, T y) \leq k \max \left\{G_{p}(x, y, y), G_{p}(x, T x, T x), G_{p}(y, T y, T y), \frac{1}{2}\left[G_{p}(x, T y, T y)+G_{p}(y, T x, T x)\right]\right\},
$$

where $k \in[0,1)$.

Suppose $T$ is a non-decreasing map such that there exists an $x_{0} \in X$ with $x_{0} \prec T x_{0}$. Also suppose that $X$ is such that for any non-decreasing sequence $\left\{x_{n}\right\}$ converging to $x, x_{n} \prec x$ for all $n \in \mathbb{N}$. Then $T$ has a fixed point. Moreover, if for each $u, v \in X$, there exists $z \in X$ which is comparable to $u$ and $v$, then $T$ has a unique fixed point. 
Proof: Observe that

$$
\begin{aligned}
& k \max \left\{G_{p}(x, y, y), G_{p}(x, T x, T x), G_{p}(y, T y, T y), \frac{1}{2}\left[G_{p}(x, T y, T y)+G_{p}(y, T x, T x)\right]\right\} \\
& =\alpha(x, y, y) G_{p}(x, y, y)+\beta(x, y, y) G_{p}(x, T x, T x)+\gamma(x, y, y) G_{p}(y, T y, T y) \\
& \quad+\delta(x, y, y)\left[G_{p}(x, T y, T y)+G_{p}(y, T x, T x)\right]
\end{aligned}
$$

where $\alpha, \beta, \gamma: X \times X \rightarrow\{0, k\}$ and $\delta: X \times X \rightarrow\left\{0, \frac{k}{2}\right\}$ are chosen such that for any $(x, y) \in X \times X$, one and only one of $\alpha(x, y, y), \beta(x, y, y), \gamma(x, y, y), \delta(x, y, y)$ is non-null. In such case,

$$
\alpha(x, y, y)+\beta(x, y, y)+\gamma(x, y, y)+2 \delta(x, y, y)=k<1
$$

Thus, the proof of the corollary follows from Theorem 2.1 .

Theorem 2.3. Let $(X, \prec)$ be a partially ordered set and suppose there exists a $G$-partial metric $G_{p}$ in $X$ such that $\left(X, G_{p}\right)$ is a complete $G$-partial metric space. Let $T: X \rightarrow X$ be a generalized Ciric quasi-contraction map such that $\alpha$ satisfies $\limsup \alpha(t)<1$ for each $a \in[0, \infty)$, for any $x, y \in X$ with $x \prec y$.

Assume that there exists $\operatorname{ann}^{a} x_{0} \in X$ with the bounded orbit, that is the sequence $\left\{x_{n}\right\}$, defined by $x_{n+1}=T x_{n}$ for all $n$, is bounded. Furthermore, if $T$ is an increasing map such that there exists an $x_{0} \in X$ with $x_{0} \prec T x_{0}$, and if any non-decreasing sequence $x_{n} \rightarrow x$ satisfies $x_{n} \prec x$ for all $n$, then $T$ has a fixed point. Moreover, if for each $x, y \in X$, there exists $z \in X$ which is comparable to $x$ and $y$, then $T$ has a unique fixed point.

Proof. Starting with $x_{0} \in X$ such that $x_{0} \prec T x_{0}$, and with T non-decreasing, we have

$$
x_{0} \prec T x_{0} \prec T^{2} x_{0} \prec T^{3} x_{0} \prec \cdots \prec T^{n} x_{0} \prec \cdots .
$$

We prove that there exists $0<c<1$ such that

$$
\alpha\left(G_{p}\left(x_{n}, x_{n+1}, x_{n+1}\right)\right)<c \text {, for each } n \geq 0 \text {. }
$$

On the contrary, assume that

$$
\lim _{k \rightarrow \infty} \alpha\left(G_{p}\left(x_{n_{k}}, x_{n_{k}+1}, x_{n_{k}+1}\right)\right)=1
$$

for some subsequence $\left\{\alpha\left(G_{p}\left(x_{n_{k}}, x_{n_{k}+1}, x_{n_{k}+1}\right)\right)\right\}$ of $\left\{\alpha\left(G_{p}\left(x_{n}, x_{n+1}, x_{n+1}\right)\right)\right\}$. Since by our assumption the sequence $\left\{G_{p}\left(x_{n}, x_{n+1}, x_{n+1}\right)\right\}$ is bounded, then the subsequence $\left\{G_{p}\left(x_{n_{k}}, x_{n_{k}+1}, x_{n_{k}+1}\right)\right\}$ is bounded too. Since the sequence is monotonic and bounded then it converges. Let $a=\lim _{k \rightarrow \infty} G_{p}\left(x_{n_{k}}, x_{n_{k}+1}, x_{n_{k}+1}\right)$. From our assumption, $\limsup _{t \rightarrow a} \alpha(t)=1$, a contradiction. Thus (8) holds.

Now, we show that $\left\{x_{n}\right\}$ is a Cauchy sequence. To prove the claim, we show by induction that for each $n \geq 2$,

$$
G_{p}\left(x_{n-1}, x_{n}, x_{n}\right) \leq K c^{n-1},
$$

where $K$ is a bound for the bounded sequence $\left\{G_{p}\left(x_{0}, x_{n}, x_{n}\right)\right\}$. When $n=2$,

$$
\begin{aligned}
G_{p}\left(T x_{0}, T^{2} x_{0}, T^{2} x_{0}\right) \leq & \alpha\left(G_{p}\left(x_{0}, T x_{0}, T x_{0}\right)\right) \max \left\{G_{p}\left(x_{0}, T x_{0}, T x_{0}\right), G_{p}\left(x_{0}, T x_{0}, T x_{0}\right),\right. \\
& \left.G_{p}\left(T x_{0}, T^{2} x_{0}, T^{2} x_{0}\right), G_{p}\left(x_{0}, T^{2} x_{0}, T^{2} x_{0}\right), G_{p}\left(T x_{0}, T x_{0}, T x_{0}\right)\right\} .
\end{aligned}
$$

From the axiom (Gp1), $G_{p}\left(T x_{0}, T x_{0}, T x_{0}\right) \leq G_{p}\left(x_{0}, T x_{0}, T x_{0}\right)$. Thus

$$
\begin{aligned}
G_{p}\left(x_{1}, x_{2}, x_{2}\right) & \leq \alpha\left(G_{p}\left(x_{0}, x_{1}, x_{1}\right)\right) \max \left\{G_{p}\left(x_{0}, x_{1}, x_{1}\right), G_{p}\left(x_{1}, x_{2}, x_{2}\right), G_{p}\left(x_{0}, x_{2}, x_{2}\right)\right\} \\
& \leq \alpha\left(G_{p}\left(x_{0}, x_{1}, x_{1}\right)\right) \max \left\{G_{p}\left(x_{0}, x_{1}, x_{1}\right), G_{p}\left(x_{0}, x_{2}, x_{2}\right)\right\} \leq K c .
\end{aligned}
$$


Thus (9) holds for $n=2$.

Suppose that (9) holds for each $k<n$; let us show that it holds for $k=n$. Since $T$ is a generalized Ciric quasicontraction map,

$$
\begin{aligned}
G_{p}\left(x_{n-1}, x_{n}, x_{n}\right) \leq & \alpha\left(G_{p}\left(x_{n-2}, x_{n-1}, x_{n-1}\right)\right) \max \left\{G_{p}\left(x_{n-2}, x_{n-1}, x_{n-1}\right), G_{p}\left(x_{n-2}, x_{n-1}, x_{n-1}\right),\right. \\
& \left.G_{p}\left(x_{n-1}, x_{n}, x_{n}\right), G_{p}\left(x_{n-2}, x_{n}, x_{n}\right), G_{p}\left(x_{n-1}, x_{n-1}, x_{n-1}\right)\right\} .
\end{aligned}
$$

From axiom (Gp1), $G_{p}\left(x_{n-1}, x_{n-1}, x_{n-1}\right) \leq G_{p}\left(x_{n-1}, x_{n}, x_{n}\right)$.

Hence (10) becomes

$$
\begin{aligned}
G_{p}\left(x_{n-1}, x_{n}, x_{n}\right) & \leq \alpha\left(G_{p}\left(x_{n-2}, x_{n-1}, x_{n-1}\right)\right) \max \left\{G_{p}\left(x_{n-2}, x_{n-1}, x_{n-1}\right), G_{p}\left(x_{n-1}, x_{n}, x_{n}\right), G_{p}\left(x_{n-2}, x_{n}, x_{n}\right)\right\} \\
& \leq \alpha\left(G_{p}\left(x_{n-2}, x_{n-1}, x_{n-1}\right)\right) \max \left\{G_{p}\left(x_{n-2}, x_{n-1}, x_{n-1}\right), G_{p}\left(x_{n-2}, x_{n}, x_{n}\right)\right\} \\
& \leq c \max \left\{G_{p}\left(x_{n-2}, x_{n-1}, x_{n-1}\right), G_{p}\left(x_{n-2}, x_{n}, x_{n}\right)\right\} .
\end{aligned}
$$

From the induction hypothesis, $G_{p}\left(x_{n-2}, x_{n-1}, x_{n-1}\right) \leq K c^{n-2}$. Thus,

$$
G_{p}\left(x_{n-1}, x_{n}, x_{n}\right) \leq c \max \left\{K c^{n-2}, G_{p}\left(x_{n-2}, x_{n}, x_{n}\right)\right\} \leq \max \left\{K c^{n-1}, c G_{p}\left(x_{n-2}, x_{n}, x_{n}\right)\right\} .
$$

We also have from the definition of $T$ and the induction hypothesis,

$$
\begin{aligned}
G_{p}\left(x_{n-2}, x_{n}, x_{n}\right) \leq & \alpha\left(G_{p}\left(x_{n-3}, x_{n-1}, x_{n-1}\right)\right) \max \left\{G_{p}\left(x_{n-3}, x_{n-1}, x_{n-1}\right), G_{p}\left(x_{n-3}, x_{n-2}, x_{n-2}\right),\right. \\
& \left.G_{p}\left(x_{n-1}, x_{n}, x_{n}\right), G_{p}\left(x_{n-3}, x_{n}, x_{n}\right), G_{p}\left(x_{n-1}, x_{n-2}, x_{n-2}\right)\right\} \\
\leq & c \max \left\{G_{p}\left(x_{n-3}, x_{n-1}, x_{n-1}\right), K c^{n-3}, G_{p}\left(x_{n-1}, x_{n}, x_{n}\right), G_{p}\left(x_{n-3}, x_{n}, x_{n}\right), K c^{n-2}\right\} \\
\leq & c \max \left\{K c^{n-3}, G_{p}\left(x_{n-3}, x_{n-1}, x_{n-1}\right), G_{p}\left(x_{n-3}, x_{n}, x_{n}\right), G_{p}\left(x_{n-1}, x_{n}, x_{n}\right)\right\} \\
\leq & \max \left\{K c^{n-2}, c G_{p}\left(x_{n-3}, x_{n-1}, x_{n-1}\right), c G_{p}\left(x_{n-3}, x_{n}, x_{n}\right), c G_{p}\left(x_{n-1}, x_{n}, x_{n}\right)\right\} .
\end{aligned}
$$

The inequality (11) becomes

$$
\begin{aligned}
G_{p}\left(x_{n-1}, x_{n}, x_{n}\right) & \leq \max \left\{K c^{n-1}, c^{2} G_{p}\left(x_{n-3}, x_{n-1}, x_{n-1}\right), c^{2} G_{p}\left(x_{n-3}, x_{n}, x_{n}\right), c^{2} G_{p}\left(x_{n-1}, x_{n}, x_{n}\right)\right\} \\
& \leq \max \left\{K c^{n-1}, c^{2} G_{p}\left(x_{n-3}, x_{n-1}, x_{n-1}\right), c^{2} G_{p}\left(x_{n-3}, x_{n}, x_{n}\right)\right\} .
\end{aligned}
$$

Repeating the same process,

$$
\begin{aligned}
G_{p}\left(x_{n-1}, x_{n}, x_{n}\right) & \leq \max \left\{K c^{n-1}, c^{3} G_{p}\left(x_{n-4}, x_{n-2}, x_{n-2}\right), c^{3} G_{p}\left(x_{n-4}, x_{n-1}, x_{n-1}\right), c^{3} G_{p}\left(x_{n-4}, x_{n}, x_{n}\right)\right\} \\
& \leq \cdots \leq \max \left\{K c^{n-1}, c^{n-1} G_{p}\left(x_{0}, x_{1}, x_{1}\right), \cdots, c^{n-1} G_{p}\left(x_{0}, x_{n}, x_{n}\right)\right\} \leq K c^{n-1} .
\end{aligned}
$$

Thus (9) holds for each $n \geq 2$. From (9) we deduce that $\left\{x_{n}\right\}$ is a Cauchy sequence.

Since $X$ is complete then there exists $q \in X$ such that $\lim _{n \rightarrow \infty} x_{n}=q$ and

$$
\lim _{n, m \rightarrow \infty} G_{p}\left(x_{n}, x_{m}, x_{m}\right)=\lim _{n \rightarrow \infty} G_{p}\left(x_{n}, q, q\right)=\lim _{n \rightarrow \infty} G_{p}\left(x_{n}, x_{n}, x_{n}\right)=G_{p}(q, q, q)=0 .
$$

Now we prove that $q$ is the fixed point of $T$. To show that, we claim that there exists $0<b<1$ such that $\alpha\left(G_{p}\left(q, x_{n}, x_{n}\right)\right)<b$.

On the contrary, we assume $\lim _{k \rightarrow \infty} \alpha\left(G_{p}\left(q, x_{n_{k}}, x_{n_{k}}\right)\right)=1$ for some subsequences $\left\{x_{n_{k}}\right\}$. Since $\lim _{k \rightarrow \infty} G_{p}\left(q, x_{n_{k}}, x_{n_{k}}\right)=0$, then $\limsup _{t \rightarrow 0} \alpha(t)=1$, a contradiction.

Since $T$ is a generalized quasi-contraction mapping we have

$$
\begin{array}{r}
G_{p}\left(T q, T x_{n}, T x_{n}\right) \leq \alpha\left(G_{p}\left(q, x_{n}, x_{n}\right)\right) \max \left\{G_{p}\left(q, x_{n}, x_{n}\right), G_{p}(q, T q, T q),\right. \\
\left.G_{p}\left(x_{n}, x_{n+1}, x_{n+1}\right), G_{p}\left(q, x_{n+1}, x_{n+1}\right), G_{p}\left(x_{n}, T q, T q\right)\right\} .
\end{array}
$$


Letting $n \rightarrow \infty$, we have, $G_{p}(T q, q, q) \leq b G_{p}(q, T q, T q)$.

Also $G_{p}(q, T q, T q) \leq b G_{p}(T q, q, q)$. Hence $G_{p}(T q, q, q) \leq b^{2} G_{p}(T q, q, q)$. Since $b<1, q=T q$.

The uniqueness of the fixed point follows from the quasicontractive condition.

Theorem 2.3 is an extension of Theorem 2.3 of Gordji et al. [4] to G-partial metric space in the sense that, if

$$
\max \left\{G_{p}(x, y, y), G_{p}(x, T x, T x), G_{p}(y, T y, T y), G_{p}(x, T y, T y), G_{p}(y, T x, T x)\right\}=G_{p}(x, y, y),
$$

in (1), then we get

$$
G_{p}(T x, T y, T y) \leq \alpha\left(G_{p}(x, y, y)\right) G_{p}(x, y, y),
$$

which is the $G$-partial metric version of the map of Gordji [9].

The proof of Corollary 2.4 follows from Theorem 2.3.

Corollary 2.4. Let $(X, \prec)$ be a partially ordered set such that there exists a $G$-partial metric on $X$ such that $\left(X, G_{p}\right)$ is a complete $G$-partial metric space. Let $T: X \rightarrow X$ be an increasing mapping such that there exists $x_{0} \in X$ with $x_{0} \prec T x_{0}$. Suppose that there exists $\alpha: \mathbb{R}^{+} \rightarrow[0,1)$ such that

$$
G_{p}(T x, T y, T y) \leq \alpha\left(G_{p}(x, y, y)\right) G_{p}(x, y, y),
$$

for all comparable $x, y \in X$. If $T$ is continuous and if for each $x, y \in X$, there exists $z \in X$ which is comparable to $x$ and $y$. Then $T$ has a unique fixed point.

Example 2.5. Let $X=\mathbb{R}^{+}$and a $G$-partial metric defined by $G_{p}(x, y, z)=\max \{x, y, z\}$ for all $x, y, z \in \mathbb{R}$. On the set $X$, we consider the usual ordering $\leq$. Clearly, $\left(X, G_{p}\right)$ is a complete $G$-partial metric space and $(X, \leq)$ is a partially ordered set. Define a function $T: \mathbb{R}^{+} \rightarrow \mathbb{R}^{+}$as follows: $T x=\frac{X}{2}$ for all $x \in \mathbb{R}$. Define $\alpha:[0, \infty) \rightarrow[0,1)$ by $\alpha(t)=\frac{t}{1+2 t}$ for each $t \in[0, \infty)$. Then we have,

$$
G_{p}(x, y, y) \leq \alpha \max \left\{G_{p}(x, y, y), G_{p}(x, T x, T x), G_{p}(y, T y, T y), G_{p}(x, T y, T y), G_{p}(y, T x, T x)\right\}
$$

for each $x, y \in X$. Thus, all of the hypotheses of Theorem 2.3 are satisfied and so $T$ has a unique fixed point (0 is the unique fixed point of $T$ ).

\section{References}

[1] Ciric, L.B. (1974) A Generalization of Banach’s Contraction Principle. Proceedings of the American Mathematical Society, 45, 267-273. http://dx.doi.org/10.2307/2040075

[2] Ciric, L.B. (1971) Generalized Contractions and Fixed Point Theorems. Publications of the Institute of Mathematics, 12, $19-26$.

[3] Wong, C.S. (1974) Generalized Contraction and Fixed Point Theorems. Proceedings of the American Mathematical Society, 42, 409-417. http://dx.doi.org/10.1090/S0002-9939-1974-0331358-4

[4] Kiany, F. and Harandi, A.A. (2013) Fixed Point Theory for Generalized Ciric Quasi-Contraction Maps in Metric Spaces. Fixed Point Theory and Applications, 2013, 6 p.

[5] Rodriguez-Lopez, R. and Nieto, J.J. (2005) Contractive Mapping Theorems in Partially Ordered Sets and Applications to Ordinary Differential Equation. A Journal on the Theory of Ordered Sets and Its Applications, 22, 223-239.

[6] Ran, A.C.M. and Reurings, M.C.B. (2003) A Fixed Point Theorem in Partially Ordered Sets and Some Applications to Matrix Equations. Proceedings of the American Mathematical Society, 132, 1435-1443. http://dx.doi.org/10.1090/S0002-9939-03-07220-4

[7] Matthews, S.G. (1992) Partial Metric Spaces. 8th British Colloquium for Theoretical Computer Science, Research Report 212, Dept. of Computer Science, University of Warwick, 708-718.

[8] Mustafa, Z. and Sims, B. (2006) A New Approach to Generalized Metric Spaces. Journal of Nonlinear and Convex Analysis, 7, 289-297.

[9] Gordji, M.E., Baghani, H. and Kim, G.H. (2012) A Fixed Point Theorem for Contraction Type Maps in Partially Ordered Metric Spaces and Application to Ordinary Differential Equations. Discrete Dynamics in Nature and Society, 2012, 981517.

[10] Saadati, R., Vaezpour, S.M., Vetro, P. and Rhoades, B.E. (2010) Fixed Point Theorems in Generalized Partially Or- 
dered G-Metric Spaces. Mathematical and Computer Modelling, 852, 797-801. http://dx.doi.org/10.1016/j.mcm.2010.05.009

[11] Turkoglu, D., Abuloha, M. and Abdejawad, T. (2011) Fixed Points of Generalized Contraction Mappings in Cone Metric Spaces. Mathematical Communications, 16, 325-334.

[12] Sastry, K.P.R. and Naidu, S.V.R. (1980) Fixed Point Theorems for Generalized Contraction Mappings. Yokohama Mathematical Journal, 28, 15-29. 